\title{
Optical spectroscopy study of the collapsed tetragonal phase of $\mathrm{CaFe}_{2}\left(\mathrm{As}_{0.935} \mathbf{P}_{0.065}\right)_{2}$ single crystals
}

\author{
X. B. Wang, ${ }^{1}$ H. P. Wang, ${ }^{1}$ T. Dong, ${ }^{1}$ R. Y. Chen, ${ }^{2}$ and N. L. Wang ${ }^{2,3,},{ }^{\circ}$ \\ ${ }^{1}$ Beijing National Laboratory for Condensed Matter Physics, \\ Institute of Physics, Chinese Academy of Sciences, Beijing 100190, China \\ ${ }^{2}$ International Center for Quantum Materials, School of Physics, Peking University, Beijing 100871, China \\ ${ }^{3}$ Collaborative Innovation Center of Quantum Matter, Beijing, China
}

\begin{abstract}
We present an optical spectroscopy study on $\mathrm{P}$-doped $\mathrm{CaFe}_{2} \mathrm{As}_{2}$ which experiences a structural phase transition from tetragonal to collapsed tetragonal (cT) phase near $75 \mathrm{~K}$. The measurement reveals a sudden reduction of low frequency spectral weight and emergence of a new feature near $3200 \mathrm{~cm}^{-1}(0.4 \mathrm{eV})$ in optical conductivity across the transition, indicating an abrupt reconstruction of band structure. The appearance of new feature is related to the interband transition arising from the sinking of hole bands near $\Gamma$ point below Fermi level in the cT phase, as expected from the density function theory calculations in combination with the dynamical mean field theory. However, the reduction of Drude spectral weight is at variance with those calculations. The measurement also indicates an absence of the abnormal spectral weight transfer at high energy (near 0.5-0.7 eV) in the $\mathrm{cT}$ phase, suggesting a suppression of electron correlation effect.

PACS numbers: 74.25.Gz, 74.70.Xa
\end{abstract}

\section{INTRODUCTION}

Understanding the relationship between electron correlations, magnetism, and unconventional superconductivity has been a focus in the current study of iron-based superconductors. Iron pnictides/chacolgenides are multi-orbital systems, different $\mathrm{Fe} 3 \mathrm{~d}$ orbitals turn out to have different fillings and band widths. $\frac{1}{2}$ The electronic correlations are also found to be orbital dependent and highly influenced by Hund's coupling. ${ }^{2-9}$ As thus, the orbital-dependent electronic correlation is of particular importance for understanding the normal state electronic properties and the pairing mechanism of the iron-based superconductors.

Experimentally, the "122" compound $\mathrm{CaFe}_{2} \mathrm{As}_{2}$ provides an excellent opportunity to study the correlation effect and its role on superconductivity as the compound appears to be easily tuned into different electronic phases. At ambient pressure $\mathrm{CaFe}_{2} \mathrm{As}_{2}$ undergoes an antiferromagentic (AFM) phase transition coupled with structural distortion at $170 \mathrm{~K}, 10$ being similar to two other 122 parent compounds $\mathrm{AFe}_{2} \mathrm{As}_{2}(\mathrm{~A}=\mathrm{Ba}$, $\mathrm{Sr}) .{ }^{11,12}$ Superconductivity could be induced by hole or electron doping. ${ }^{13-17}$ On the other hand, it is found that $\mathrm{CaFe}_{2} \mathrm{As}_{2}$ is rather sensitive to pressure ${ }^{18-22}$ With hydrostatic pressure, it undergoes a structural transition from a tetragonal ( $\mathrm{T}$ ) phase to a collapsed tetragonal (cT) phase..$^{21}$ The cT phase has the same crystal symmetry as the high-temperature $\mathrm{T}$ phase but with an abrupt $\sim 10 \%$ reduction in c-axis parameters and an $2 \%$ increase along the a-axis. The cT phase can also be stabilized at ambient pressure by chemical substitution on As site, 23 Fe site, 16 and Ca site..$^{24.25}$

The $\mathrm{T}$ to $\mathrm{cT}$ phase transition is linked with the formation of direct As-As interlayer bond and a weakening of in-plane FeAs bonds ${ }^{21,26}$. Magnetic and electronic structures are found to be strongly influenced by the transition. In the cT phase, the Fe local moment is quenched ${ }^{27,28}$, the AFM order or magnetic fluctuation disappears ${ }^{29-31}$ and the standard Fermi liquid behavior recovers. ${ }^{16,23}$ In addition, in sharp contrast to the paramagnetism in the $\mathrm{T}$ phase, intrinsic superconductivity is absent in the cT phase. Recent angle-resolved photoemission spectroscopy (ARPES) measurements show that the two electron pockets at the $M$ point transform into one cylinder, and the hole pockets at $\Gamma$ disappear, $32-34$ being in agreement with the band structure calculations. ${ }^{23,35,36}$ However, in a de Haasvan Alphen effect study on $\mathrm{CaFe}_{2} \mathrm{P}_{2}$, which is a structural analogue of the cT phase of $\mathrm{CaFe}_{2} \mathrm{As}_{2}$, the hole pockets are not completely vanished, two small hole pockets centered at $\mathrm{Z}$ point still exist 37 Very recently, two density function theory (DFT) calculations in combination with the dynamical mean field theory (DMFT) (DFT-DMFT) have been performed on both $\mathrm{T}$ and $\mathrm{cT}$ phases in an effort to address the electron correlation and its effect on electronic properties 7.8 . It is found that the electron correlation becomes weaker for all Fe $3 \mathrm{~d}$ orbitals in cT phase. Especially, the Fe $3 \mathrm{~d}_{x y}$ orbital undergoes a change from being the most strongly renormalized orbital in the $\mathrm{T}$ phase to the least renormalized orbital in the $\mathrm{cT}$ phase ${ }^{8}$. The calculated optical conductivity indicates a kinetic energy gain due to the loss in Hund's rule coupling energy in the cT phase. In addition, a new feature near $0.5 \mathrm{eV}$ emerges in the optical conductivity ${ }^{7}$.

It is highly desirable to investigate experimentally the charge dynamics in the cT phase and to compare with the DFT-DMFT calculations. In this work, we present a transport and an optical spectroscopy study of the collapsed tetragonal phase of $\mathrm{CaFe}_{2}\left(\mathrm{As}_{0.935} \mathrm{P}_{0.065}\right)_{2}$ single crystals. The isovalent substitution of As with $\mathrm{P}$ in $\mathrm{CaFe}_{2} \mathrm{As}_{2}$ does not introduce extra carriers, but induce a structural transition to the c $\mathrm{T}$ phase near $75 \mathrm{~K}$. An interfacial or filamentary superconductivity at about $30 \mathrm{~K}$ emerges at the same time. Our optical spectroscopy measurement reveals a sudden spectral change at low frequencies, indicating an abrupt reconstruction of band structure. A new feature appears near $0.4 \mathrm{eV}$, being roughly consistent with the DFT-DMFT calculations. However, the overall Drudelike spectral weight is lowered in the cT phase. We analyze the low-frequency data in terms of a Drude-Lorentz approach containing a narrow and a broad Drude components. On the other hand, the spectral weight transfer at high energy, commonly observed for $\mathrm{AFe}_{2} \mathrm{As}_{2}(\mathrm{~A}=\mathrm{Ba}, \mathrm{Sr}, \mathrm{Ca})$, is absent in the cT phase, which could be assigned to the weakening of the 
Hund's coupling energy. The sudden weakening of electron correlation appears to be correlated to the vanishing of intrinsic superconductivity in the collapse cT phase.

\section{EXPERIMENT}

High quality of $\mathrm{CaFe}_{2}\left(\mathrm{As}_{1-x} \mathrm{P}_{x}\right)_{2}$ single crystals with nominal composition of $\mathrm{x}=0.065$ were grown by the self-flux method. The typical size was about $5 \times 5 \times 0.1 \mathrm{~mm}^{3}$. The actual $\mathrm{x}$ values was about $0.0575 \pm 0.008$ determined by energy dispersion X-rays spectrum (EDX) on several pieces of samples in the same batch. Resistivity measurements were performed with a Quantum Design Physical Property Measurement System (PPMS). Magnetization was measured using a Quantum Design superconducting quantum interference device (SQUID-VSM). The optical reflectance measurements were performed on Bruker IFS 113v and 80v spectrometers in the frequency range from 50 to $35000 \mathrm{~cm}^{-1}$. An in situ gold and aluminum overcoating technique was used to obtain the reflectivity $\mathrm{R}(\omega)$. The real part of conductivity $\sigma_{1}(\omega)$ is obtained by the Kramers-Kronig transformation of $R(\omega)$. The Hagen-Rubens relation was used for low frequency extrapolation; at high frequency side a $\omega^{-1}$ relation was used up to 300 $000 \mathrm{~cm}^{-1}$, above which $\omega^{-4}$ was applied.

\section{RESULTS AND DISCUSSION}

Figure 1 shows the temperature dependence of in-plane resistivity for $\mathrm{CaFe}_{2}\left(\mathrm{As}_{1-x} \mathrm{P}_{x}\right)_{2}$ single crystals at zero field. In the case of $\mathrm{x}=0$, the parent $\mathrm{CaFe}_{2} \mathrm{As}_{2}$ shows a sharp increase in resistivity at $\mathrm{T}_{N} \approx 165 \mathrm{~K}$, corresponding to a firstorder structure transition from the tetragonal phase to the orthorhombic AFM phase. By substituting isovalent P for As, the SDW state is suppressed and an abrupt decrease of resistivity occurs at about $\mathrm{T}_{c T} \approx 75 \mathrm{~K}$ upon cooling. The large

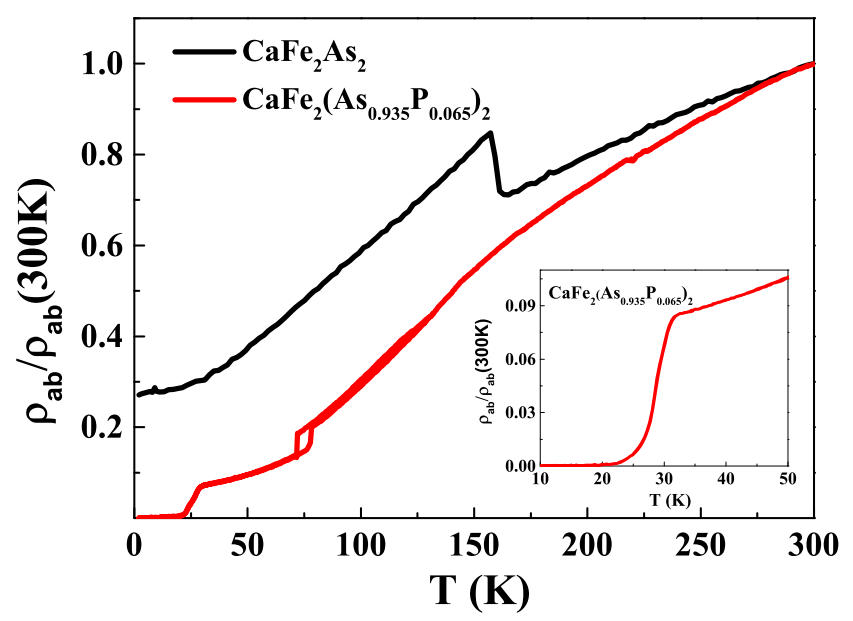

FIG. 1: (Color online) Temperature-dependence of normalized resistivity of $\mathrm{CaFe}_{2} \mathrm{As}_{2}$ and $\mathrm{CaFe}_{2}\left(\mathrm{As}_{0.935} \mathrm{P}_{0.065}\right)_{2}$. The inset plots an enlarged region for superconducting transition in $\mathrm{CaFe}_{2}\left(\mathrm{As}_{0.935} \mathrm{P}_{0.065}\right)_{2}$.

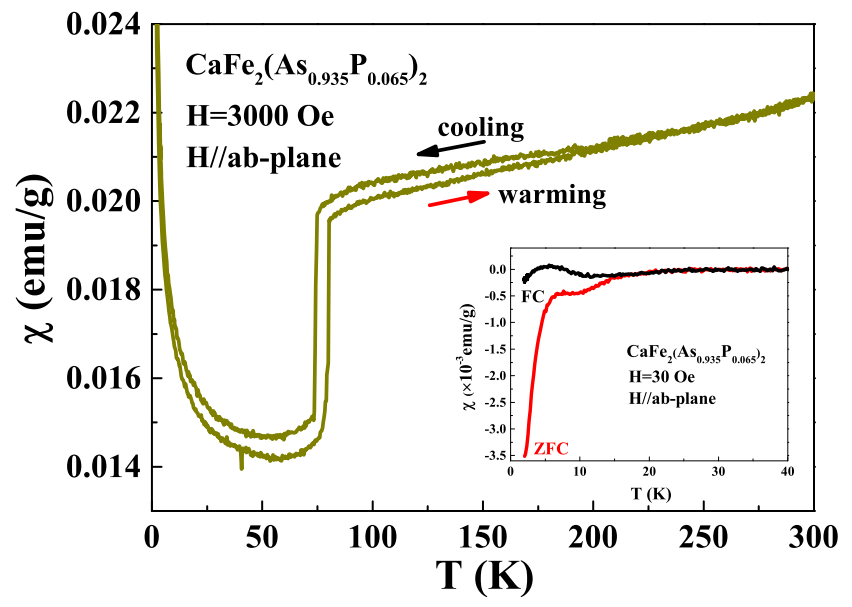

FIG. 2: (Color online) Temperature-dependence of magnetic susceptibility of $\mathrm{CaFe}_{2}\left(\mathrm{As}_{0.935} \mathrm{P}_{0.065}\right)_{2}$ with $\mathbf{H} \| a b$-plane at $3000 \mathrm{Oe}$. The inset shows the zero-field-cooled and field-cooled data at $30 \mathrm{Oe}$.

thermal hysteresis about $10 \mathrm{~K}$ suggest the first-order nature of the transition. With a further decreasing in temperature, the resistivity shows a $\mathrm{T}^{2}$ dependence, which indicates the recovery of Fermi liquid behavior, consistent with the previous reports $\frac{16,23}{1}$ Notably, there is another obvious down-turn at about $32 \mathrm{~K}$, and the resistivity reaches zero at about $20 \mathrm{~K}$, as shown in the inset of Fig. 1. This is very similar to the case of rare-earth-doped $\mathrm{CaFe}_{2} \mathrm{As}_{2}$, where the superconductivity is considered as an interfacial or filamentary effect. This is very interesting because it was suggested that isovalent substitution of $\mathrm{P}$ for $\mathrm{As}$ in $\mathrm{CaFe}_{2} \mathrm{As}_{2}$ couldn't induce superconductivity before. ${ }^{23}$

The temperature dependence of dc susceptibility of $\mathrm{CaFe}_{2}\left(\mathrm{As}_{0.935} \mathrm{P}_{0.065}\right)_{2}$ single crystals in an applied magnetic filed of $\mathrm{H}=0.3 \mathrm{~T}$ aligned in ab-plane is shown in Fig. 2. The susceptibility decreases slightly with cooling temperatures, but drops abruptly below $\mathrm{T}_{c T}$. The strong Curie-Weisslike tail at lower temperature is not intrinsic and can be attributed to the presence of paramagnetic impurities or defects, since Knight shift measurements on compounds showing similar Curie-Weiss behavior in the cT phase did not reveal any upturn. ${ }^{29}$ The huge thermal hysteresis and sudden decrease of susceptibility provides further evidence of first-order transition in this compound. At $30 \mathrm{Oe}$, as shown the inset of Fig. 2 , a diamagnetic signal of superconducting transition at about $15 \mathrm{~K}$, and a rapid drop of the dc susceptibility at $7 \mathrm{k}$ are observed. This is very similar to $\mathrm{Pr}$-doped $\mathrm{CaFe}_{2} \mathrm{As}_{2}$, suggesting the possible existence of two superconducting phases ${ }^{38}$ However as shown in the Fig. 2, the superconductivity is killed at a small magnetic filed $(\mathrm{H}=0.3 \mathrm{~T})$, consistent with interfacial or filamentary superconductivity scenario. Both the resistivity and magnetic properties suggest that the SDW state is supressed and the system exhibits a first-order structural phase transition from tetragonal to collapsed-tetragonal phase in $\mathrm{CaFe}_{2}\left(\mathrm{As}_{0.935} \mathrm{P}_{0.065}\right)_{2}$ single crystals.

Figure 3(a) and (b) show the reflectance spectra of the $\mathrm{CaFe}_{2}\left(\mathrm{As}_{1-x} \mathrm{P}_{x}\right)_{2}$ crystals $(\mathrm{x}=0$ and 0.065$)$ at different temperatures. The reflectance exhibits a metallic response in both 

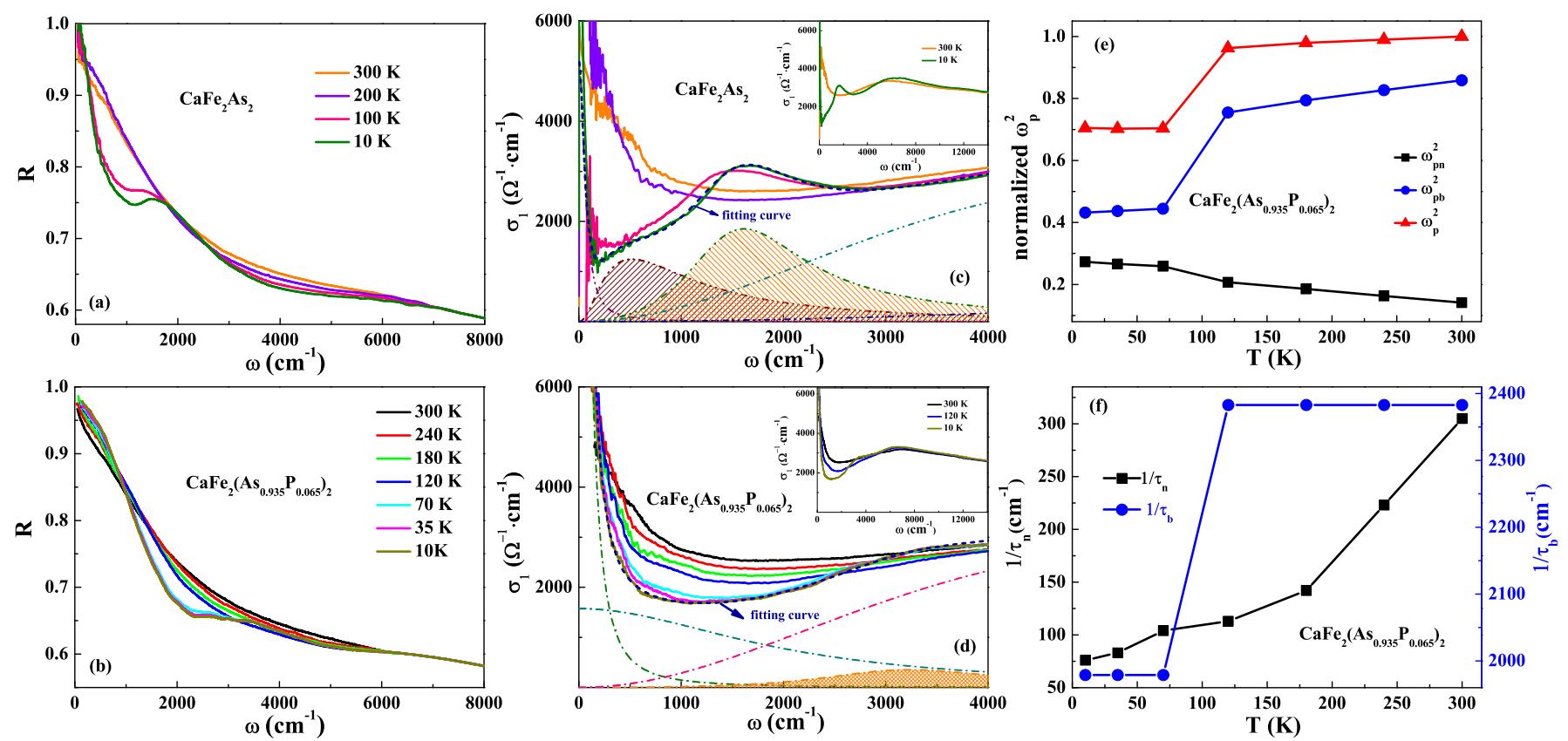

FIG. 3: (Color online). Left panel: $\mathrm{R}(\omega)$ for (a) $\mathrm{CaFe}_{2} \mathrm{As}_{2}$ and (b) $\mathrm{CaFe}_{2}\left(\mathrm{As}_{0.935} \mathrm{P}_{0.065}\right)_{2}$ below $8000 \mathrm{~cm}^{-1}$. Middle panel: $\sigma_{1}(\omega)$ for (c) $\mathrm{CaFe}_{2} \mathrm{As}_{2}$ and (d) $\mathrm{CaFe}_{2}\left(\mathrm{As}_{0.935} \mathrm{P}_{0.065}\right)_{2}$ blow $4000 \mathrm{~cm}^{-1}$. The Drude-Lorentz fit for $\mathrm{T}=10 \mathrm{~K}$ are shown at the bottom. Inset: the optical conductivity over a much wider frequency range. Right panel: Temperature dependence of $\omega_{p}^{2}$ normalized to the value of $300 \mathrm{~K}$ and scattering rate $1 / \tau$ of the two Drude terms of $\mathrm{CaFe}_{2}\left(\mathrm{As}_{0.935} \mathrm{P}_{0.065}\right)_{2}$.

frequency and temperature dependence, and the most obvious feature is the suppression of $\mathrm{R}(\omega)$ in both compounds at low temperatures. As a consequence, relatively sharp reflectance edges appear at low frequency, which suggests a reduction of the carrier scattering rate. In the parent $\mathrm{CaFe}_{2} \mathrm{As}_{2}$, whose behavior is very similar to that of the other two members of

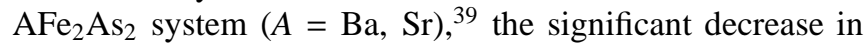
energy scale of the reflectance edge implies a considerable reduction of carrier density. While in the P-doped $\mathrm{CaFe}_{2} \mathrm{As}_{2}$, there is a sudden suppression of the reflectance between 1000 $\mathrm{cm}^{-1}$ to $3500 \mathrm{~cm}^{-1}$ across the T to cT phase transition. As a result, the spectra were separated into two groups above and below $\mathrm{T}_{c T}$, suggesting an abrupt band reconstruction as well. Nevertheless, the spectral change is distinctly different from the AFM phase for the parent compound.

The middle panels of Fig. 3 show the conductivity spectra $\sigma_{1}(\omega)$ below $4000 \mathrm{~cm}^{-1}$. In the parent compound, the spectra is severely suppressed at low frequencies, only a very sharp and narrow Drude term is left, and a double-peak feature appears blow $\mathrm{T}_{N}$ which are known as the SDW gaps ${ }^{39}$ as shown in the Fig. 3 (c). While in the P-doped compound, the optical conductivity is suppressed suddenly below $\mathrm{T}_{C T}$ at much higher and wider frequency range. A peak-like structure appears near $3200 \mathrm{~cm}^{-1}(0.4 \mathrm{eV})$, which we shall discuss later. The data are in agreement with a recent DFT-DFMT calculation of the collapsed tetragonal phase of $\mathrm{CaFe}_{2} \mathrm{As}_{2}{ }^{\frac{7}{7}}$

To make a quantitative analysis of the temperature evolution of the different part of electronic excitations, we decompose the optical conductivity using a simple Drude-lorentz model: ${ }^{39}$

$$
\epsilon(\omega)=\epsilon_{\infty}-\sum_{i} \frac{\omega_{p, i}^{2}}{\omega_{i}^{2}+i \omega / \tau_{i}}+\sum_{i} \frac{\Omega_{i}^{2}}{\omega_{i}^{2}-\omega^{2}-i \omega / \tau_{i}} .
$$

where $\epsilon_{\infty}$ is the dielectric constant at high energy, and the middle and last terms are the Drude and Lorentz components, respectively. The Drude components represent the contribution from conduction electrons, while the Lorentz components describe the interband transitions.

We are mainly concerned about the evolution of the lowenergy optical conductivity. Unlike the parent $\mathrm{CaFe}_{2} \mathrm{As}_{2}$, the low-energy optical conductivity of $\mathrm{CaFe}_{2}\left(\mathrm{As}_{0.935} \mathrm{P}_{0.065}\right)_{2}$ can fit very well by two Drude terms, a narrow one and a broad one, at all temperatures as shown in Fig. 3(d). The two Drude components may provide information about different types of carriers in this compound. ${ }^{40}$ The temperature dependence of normalized plasma frequency $\omega_{p}^{2}$ and scattering rate $1 / \tau$ are shown in Fig. 3 (e) and (f). The subscripts $n$ and $b$ stand for the narrow and broad Drude terms, respectively.

The plasma frequency $\omega_{p, b}^{2}$ undergoes a discontinuous decrease at $\mathrm{T}_{c T}$, however $\omega_{p, n}^{2}$ shows an opposite trend. Nevertheless, the total low energy plasma frequency $\omega_{p}^{2}=\omega_{p, n}^{2}+\omega_{p, b}^{2}$ decreases by about $20 \%$ in the cT phase, indicating an overall decrease of $\mathrm{n} / \mathrm{m}^{*}$. Very recently, the angle resolved photoemission spectroscopy (ARPES) investigations on the cT phase of $\mathrm{CaFe}_{2} \mathrm{As}_{2}$ single crystals suggest that the hole pockets near $\Gamma$ point sink below the Fermi level while the electron band moves to a higher binding energy, resulting in a decrease of the band effective mass of electron pocket ${ }^{32-34}$ Evidently, if 
we associate the narrow Drude component with the electron pocket and the broad one with the hole pocket, we can attribute the increase of $\omega_{p, n}^{2}$ to the reduction of band mass of electron pocket and the decrease of $\omega_{p, b}^{2}$ to the partial removal of hole pocket (mainly close to $\Gamma$ point). In an earlier study on various $3 \mathrm{~d}$ transition metal based 122 compounds, Cheng et al. found that the moderate $3 \mathrm{~d}$-electron correlation is responsible for the incoherent term (the broad Drude term here) in the iron pnictides. ${ }^{41}$ Thus the decrease of $\omega_{p, b}^{2}$ suggests a suppression of correlation effect, which is consistent with recent NMR and theoretical study, ${ }^{7,29}$ It deserves to remark that, although the effective mass from electron Fermi surface is indeed reduced in the cT phase, $\stackrel{7,33}{,}$ the overall plasma frequency $\omega_{p}^{2}$ is still reduced due to the large reduction of hole carrier density. Since the kinetic energy of the electrons is proportional to the plasma frequency, the measurement indicates that the overall kinetic energy of charge carriers decreases in the cT phase. The result is different from the DFT-DMFT calculations that suggests an increase of the low frequency spectral weight in the cT phase. We notice that, in the above-mentioned calculations, the optical kinetic energy was estimated by summarizing the conductivity spectrum up to a certain cutoff frequency, and the same cutoff frequency was used for both $\mathrm{T}$ and $\mathrm{cT}$ phases. In fact, the Drude spectral weight is shifted to lower frequency in $\mathrm{cT}$ phase in comparison with $\mathrm{T}$ phase. Therefore, different cutoff frequencies should be used in summarizing the Drude components of conductivity for the two phases. Further work is needed to address this issue.

Figure 3 (f) shows the $\mathrm{T}$ dependence of the scattering rate of the two Drude components. The scattering rate of the narrow Drude term $1 / \tau_{n}$ decreases upon cooling and there is a downturn below $\mathrm{T}_{c T}$, while the $1 / \tau_{b}$ decreases suddenly below $\mathrm{T}_{c T}$. In the optimally doped $\mathrm{K}$ - and P-doped $\mathrm{BaFe}_{2} \mathrm{As}_{2}, 1 / \tau_{n}$ decreases linearly with temperature and $1 / \tau_{b}$ keeps constant in the whole temperature range above $\mathrm{T}_{c}{ }^{42}$. Then, the sudden changes of $1 / \tau_{n}$ and $1 / \tau_{b}$ in $\mathrm{CaFe}_{2}\left(\mathrm{As}_{0.935} \mathrm{P}_{0.065}\right)_{2}$ upon entering the cT phase may be related to the suppression of spin fluctuation or the reduction of scattering channel caused by the loss of hole pockets near $\Gamma$ point.

To further elaborate on the spectral evolution, we plot the frequency-dependent integrated spectral weight of $\mathrm{CaFe}_{2}\left(\mathrm{As}_{1-x} \mathrm{P}_{x}\right)_{2}(\mathrm{x}=0$ and 0.065$)$ at $10 \mathrm{~K}$, as shown in Fig. 4 , and the low-temperature integrated spectral weight has been normalized to the integrated spectral weight at room temperature. For the parent $\mathrm{CaFe}_{2} \mathrm{As}_{2}$, the strong dip blow 1000 $\mathrm{cm}^{-1}$ represents the formation of the SDW gaps, and the small turn point around $4000 \mathrm{~cm}^{-1}$ is attributed to unconventional spectral weight transformation due to Hund's coupling. ${ }^{43}$ In the P-doped compound, there is no SDW gap that develops at low temperature, and the low- $\omega$ spectral weight change is induced simply by the narrowing of Drude component. Additionally, a new peak emerges starting from $3000 \mathrm{~cm}^{-1}$, leading to a gradual recovery of the spectral weight at very high energy. As we have mentioned, the hole bands at $\Gamma$ point, which cross the Fermi level above $\mathrm{T}_{c T}$, suddenly sink below the Fermi level in the $\mathrm{cT}$ phase, as a consequences, the original intraband transition in the $\mathrm{T}$ phase would vanish in the $\mathrm{cT}$ phase and an extra interband transition around $\Gamma$ point would

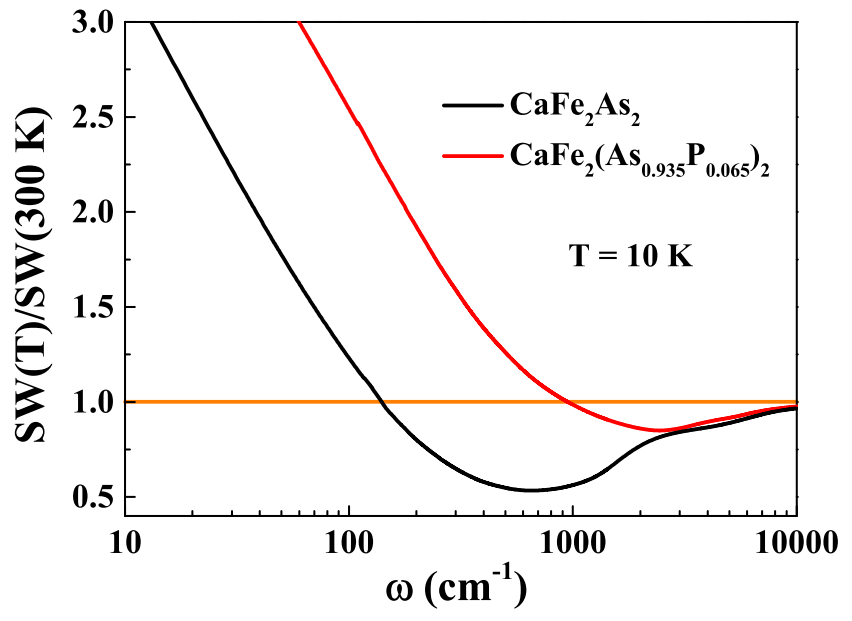

FIG. 4: (Color online). Ratio of the integrated spectral weight as a function of $\omega$ at $10 \mathrm{~K}$ in $\mathrm{CaFe}_{2} \mathrm{As}_{2}$ and $\mathrm{CaFe}_{2}\left(\mathrm{As}_{0.935} \mathrm{P}_{0.065}\right)_{2}$. The low-temperature integrated spectral weight is normalized to the room-temperature data.

appear. The structure is also evident in the DFT-DMFT calculations but with the peak centered at slightly higher energy $0.5 \mathrm{eV}^{7}$

Finally, we'd like to comment shortly on the spectra at higher energies. It is well established that, for the ironpnictides/chacogenides, there exist a temperature-induced spectral weight transfer at high energies. With decreasing temperature, the low-frequency spectral weight is transferred to the high-energy region (usually above $0.5 \sim 0.7 \mathrm{eV}$ ) ${ }^{39.43-45}$. Such spectral weight transfer was ascribed to the electron correlation effect, in particular, to the Hund's coupling effect between itinerant Fe $3 \mathrm{~d}$ electrons and localized Fe $3 \mathrm{~d}$ electrons in different orbitals 43,44 . It represents the redistribution of the spectral weight between different $3 d$ bands ${ }^{43,45}$. In the present $\mathrm{P}$-doped sample, this temperature dependent spectral weight transfer at high energy is not visible in the cT phase, as shown in the inset of middle pinel of Fig. 3. It provides further evidence that the electron correlation is suppressed in the cT phase. The result is also consistent with the DFTDMFT calculations which indicates a loss of Hund's coupling energy. The suppression of the electron correlations, including the Hund's coupling, is apparently linked with the loss of Fe magnetic moment and disappearance of superconductivity in the cT phase.

\section{SUMMARY}

In summary, a systematic investigation of resistivity, susceptibility, and optical spectroscopy is performed on the collapsed tetragonal phase of $\mathrm{CaFe}_{2}\left(\mathrm{As}_{0.935} \mathrm{P}_{0.065}\right)_{2}$ single crystals. The isovalent substitution of $\mathrm{P}$ for As suppresses the SDW state and results in a first-order structural phase transition from tetragonal to collapsed-tetragonal phase. It also induces an interfacial or filamentary superconductivity near 30 $\mathrm{K}$. Related to the change in the topology of the Fermi surface, 
the optical spectroscopy shows a sudden change. The lowenergy optical spectrum can be decomposed into two components, a broad and a narrow Drude in the whole temperature range. The narrow Drude term increases its spectral weight in the cT phase which can be attributed to the decrease of the band effective mass of electron pocket, whereas the weight of broad Drude term decreases abruptly being likely due to the vanish of the hole pockets near $\Gamma$ point, which also leads to an additional mid-infrared peak in the cT phase. Furthermore, our data reveal that the electron correction is suppressed, which is correlated with the loss of Fe magnetic mo- ment and quenching of superconductivity in the $\mathrm{cT}$ phase.

\section{Acknowledgments}

We acknowledge useful discussions with Subhasish Mandal. This work is supported by the National Science Foundation of China (11120101003, 11327806), and the 973 project of the Ministry of Science and Technology of China (2011CB921701, 2012CB821403).
* Electronic address: nlwang@pku.edu.cn

1 D. H. Lu, M. Yi, S.-K. Mo, A. S. Erickson, J. Analytis, J.-H. Chu, D. J. Singh, Z. Hussain, T. H. Geballe, I. R. Fisher and Z.-X. Shen, Nature 455, 81 (2008).

2 K. Haule and G. Kotliar, New Journal of Physics 11, 025021 (2009).

3 H. Ishida and A. Liebsch, Phys. Rev. B 81, 054513 (2010).

4 Wei-Guo Yin, Chi-Cheng Lee, and Wei Ku, Phys. Rev. Lett. 105, 107004 (2010)

5 L. de' Medici, Phys. Rev. B 83, 205112 (2011).

${ }^{6}$ R. Yu and Q. Si, Phys. Rev. B 86, 085104 (2012).

7 Subhasish Mandal, R. E. Cohen, and K. Haule, Phys. Rev. B 90, 060501(R) (2014).

8 J. Diehl, S. Backes, D. Guterding, H. O. Jeschke, and R. Valenti, Phys. Rev. B 90, 085110 (2014).

9 M. J. Calderon, L. de' Medici, B. Valenzuela, and E. Bascones, arXiv:1407.6935

10 N. Ni, S. Nandi, A. Kreyssig, A. I. Goldman, E. D. Mun, S. L. Bud'ko, and P. C. Canfield, Phys. Rev. B 78, 014523 (2008).

11 M. Rotter, M. Tegel, D. Johrendt, I. Schellenberg, W. Hermes, and R. Pottgen, Phys. Rev. B 78, 020503(R) (2008).

12 J.-Q. Yan, A. Kreyssig, S. Nandi, N. Ni, S. L. Bud'ko, A. Kracher, R. J. McQueeney, R. W. McCallum, T. A. Lograsso, A. I. Goldman, and P. C. Canfield, Phys. Rev. B 78, 024516 (2008).

13 P. M. Shirage, K. Miyazawa, H. Kito, H. Eisaki and A. Iyo, Appl. Phys. Express 1, 081702 (2008).

14 D. M. Wang, X. C. Shangguan, J. B. He, L. X. Zhao, Y. J. Long, P. P. Wang, and L. Wang, J. Supercond. Nov. Magn. 26, 2121 (2013).

15 N. Kumar, R. Nagalakshmi, R. Kulkarni, P. L. Paulose, A. K. Nigam, S. K. Dhar, and A. Thamizhavel, Phys. Rev. B 79, 012504 (2009).

${ }^{16}$ M. Danura, K. Kudo, Y. Oshiro, S. Araki, T. C. Kobayashi, and M. Nohara: J. Phys. Soc. Jpn. 80, 103701 (2011).

17 S. R. Saha, N. P. Butch, T. Drye, J. Magill, S. Ziemak, K. Kirshenbaum, P. Y. Zavalij, J. W. Lynn, and J. Paglione, Phys. Rev. B 85, 024525 (2012).

18 M. S. Torikachvili, S. L. Bud'ko, N. Ni, and P. C. Canfield, Phys. Rev. Lett. 101, 057006 (2008).

19 T. Park, E. Park, H. Lee, T. Klimczuk, E. D. Bauer, F. Ronning and J. D. Thompson, J. Phys. Condens. Matter 20, 322204 (2008).

${ }^{20}$ W. Yu, A. A. Aczel, T. J. Williams, S. L. Bud'ko, N. Ni, P. C. Canfield, and G. M. Luke, Phys. Rev. B 79, 020511 (2009).

${ }^{21}$ A. Kreyssig, M. A. Green, Y. B. Lee, G. D. Samolyuk, P.Zajdel, J.W. Lynn, S. L. Budko, M. S. Torikachvili, N. Ni, S. Nandi, J. B. Leao, S. J. Poulton, D. N. Argyriou, B. N. Harmon, R. J. McQueeney, P. C. Canfield, and A. I. Goldman, Phys. Rev. B 78, 184517 (2008).
22 A. I. Goldman, A. Kreyssig, K. Prokes, D. K. Pratt, D. N. Argyriou, J. W. Lynn, S. Nandi, S. A. J. Kimber, Y. Chen, Y. B. Lee, G. Samolyuk, J. B. Leao, S. J. Poulton, S. L. Budko, N. Ni, P. C. Canfield, B. N. Harmon, and R. J. McQueeney, Phys. Rev. B 79, 024513 (2009).

${ }^{23}$ S. Kasahara, T. Shibauchi, K. Hashimoto, Y. Nakai, H. Ikeda, T. Terashima, and Y. Matsuda, Phys. Rev. B 83, 060505 (2011).

${ }^{24}$ S. R. Saha, N. P. Butch, T. Drye, J. Magill, S. Ziemak, K. Kirshenbaum, P. Y. Zavalij, J. W. Lynn, and J. Paglione, Phys. Rev. B 85, 024525 (2012).

25 J. R. Jeffries, N. P. Butch, K. Kirshenbaum, S. R. Saha, G. Samudrala, S. T. Weir, Y. K. Vohra, and J. Paglione, Phys. Rev. B 85, 184501 (2012).

26 T. Yildirim, Phys. Rev. Lett. 102, 037003 (2008).

27 H. Gretarsson, S. R. Saha, T. Drye, J. Paglione, Jungho Kim, D. Casa, T. Gog, W. Wu, S. R. Julian, and Young-June Kim, Phys. Rev. Lett. 110, 047003 (2013).

${ }^{28}$ L. Ma, G.-F. Ji, J. Dai, S. R. Saha, T. Drye, J. Paglione, and W.-Q. Yu, Chin, Phys. B 22, 057401 (2013).

29 Y. Furukawa, B. Roy, S. Ran, S. L. Budko, and P. C. Canfield, Phys. Rev. B 89, 121109(R) (2014).

${ }^{30}$ D. K. Pratt, Y. Zhao, S. A. J. Kimber, A. Hiess, D. N. Argyriou, C. Broholm, A. Kreyssig, S. Nandi, S. L. Budko, N. Ni, P. C. Canfield, R. J. McQueeney, and A. I. Goldman, Phys. Rev. B 79, 060510 (2009).

31 J. H. Soh, G. S. Tucker, D. K. Pratt, D. L. Abernathy, M. B. Stone, S. Ran, S. L. Budko, P. C. Canfield, A. Kreyssig, R. J. McQueeney, and A. I. Goldman, Phys. Rev. Lett. 111, 227002 (2013).

32 K. Gofryk, B. Saparov, T. Durakiewicz, A. Chikina, S. Danzenbacher, D. V. Vyalikh, M. J. Graf, and A. S. Sefat, Phys. Rev. Lett. 112, 186401 (2014).

${ }^{33}$ R. S. Dhaka, Rui Jiang, S. Ran, S. L. Bud'ko, P. C. Canfield, B. N. Harmon, Adam Kaminski, Milan Tomic, Roser Valenti, and Yongbin Lee, Phys. Rev. B 89, 020511(R) (2014).

34 Koji TSUBOTA, Takanori WAKITA, Hiroki NAGAO, Chiaki HIRAMATSU, Toshihiko ISHIGA, Masanori SUNAGAWA, Kanta ONO, Hiroshi KUMIGASHIRA, Masataka DANURA, Kazutaka KUDO, Minoru NOHARA, Yuji MURAOKA, and akayoshi YOKOYA, J. Phys. Soc. Jpn. 82073705 (2013).

35 Yu-Zhong Zhang, Hem C. Kandpal, Ingo Opahle, Harald O. Jeschke, and Roser Valenti, Phys. Rev. B 80, 094530(R) (2009).

36 Milan Tomic, Roser Valenti, and Harald O. Jeschke, Phys. Rev. B 85, 094105 (2012).

37 Amalia I. Coldea, C. M. J. Andrew, J. G. Analytis, R. D. McDonald, A. F. Bangura, J.-H. Chu, I. R. Fisher, and A. Carrington, Phys. Rev. Lett. 103, 026404 (2009). 
38 B. Lv, L. Deng, M. Gooch, F. Wei, Y. Sun, J. K. Meen, Y.-Y. Xue, B. Lorenz, and C.-W. Chu, Proc. Natl. Acad. Sci. U.S.A. 108, 15705 (2011).

${ }^{39}$ W. Z. Hu, J. Dong, G. Li, Z. Li, P. Zheng, G. F. Chen, J. L. Luo, and N. L. Wang, Phys. Rev. Lett. 101, 257005 (2008).

40 J. J. Tu, J. Li, W. Liu, A. Punnoose, Y. Gong, Y. H. Ren, L. J. Li, G. H. Cao, Z. A. Xu, and C. C. Homes, Phys. Rev. B 82, 174509 (2010).

41 B. Cheng, B. F. Hu, R. Y. Chen, G. Xu, P. Zheng, J. L. Luo, and N. L. Wang, Phys. Rev. B 86, 134503 (2012).
${ }^{42}$ Y. M. Dai, B. Xu, B. Shen, H. Xiao, H. H. Wen, X. G. Qiu, C. C. Homes, and R. P. S. M. Lobo, Phys. Rev. L 111, 117001 (2013).

${ }^{43}$ N. L. Wang, W. Z. Hu, Z. G. Chen, R. H. Yuan, G. Li, G. F. Chen and T. Xiang, J. Phys.: Condens. Matter 24294202 (2012).

${ }^{44}$ A. A. Schafgans, S. J. Moon, B. C. Pursley, A. D. LaForge, M. M. Qazilbash, A. S. Sefat, D. Mandrus, K. Haule, G. Kotliar, and D. N. Basov, Phys. Rev. Lett. 108, 147002 (2012).

${ }^{45}$ R. H. Yuan, W. D. Kong, L. Yan, H. Ding, and N. L. Wang, Phys. Rev. B 87, 144517 (2013). 